\title{
Guided surgery technique - review of accuracy and errors
}

\author{
Sorin Nicolae Popescu, Gabriel Ciochinda, Mihai Burlibasa, Gabriela Tanase, \\ Augustin Mihai, Viorel Stefan Perieanu, Madalina Violeta Perieanu, Irina Donciu, \\ Oana Cella Andrei, Corina Marilena Cristache, Ioana Voinescu, \\ Ileana Ionescu, Luminita Oancea \\ "Carol Davila" University of Medicine and Pharmacy, Bucharest, Romania
}

\begin{abstract}
Data on the functional three-dimensional positioning of the future restoration are important for planning a prosthetic guided insertion of the dental implants. Errors can occur in the digital and analogous process of analysis, design and production of the surgical guides, possibilities of minimizing them being vital for obtaining a predictable surgical and prosthetic process.
\end{abstract}

Keywords: implant-aided surgery, predictability, error

\section{INTRODUCTION}

Virtual planning for exact placement of dental implants can now be used in a fluent process using computer tomography, (CT), virtual planning, optical scanning, virtual computed added design (CAD), printing and milling $(1,2)$. Guided implantology has made possible to reduce the time of surgery (3), to apply techniques without taking off the flap (flapless procedure), to reduce pain, to reduce post-surgery inflammation and immediate temporary prosthesis (4).

For a predictable implant-prosthetic rehabilitation, it is not sufficient to place the implant in the area where there is enough bone volume, it is necessary to have data on the functional three-dimensional positioning of the future restoration so that a prosthetic guided insertion of the dental implants is performed (5). The use of pre-surgical planning in the design software allows, in addition to the analysis of the height and thickness of the edentulous ridge, to quantify the volume of bone addition needed, to choose the type of optimal prosthetic restoration and the proper connection at the implant level (cementation versus screwing).

The insertion guides of the implants obtained by milling or printing, ensure also the precise placement of the dental implants from the point of view of the drilling depth, avoiding the damage caused at the level of the mandibular nerves, the mental foramen and the maxillary sinuses. Due to the proximity of these structures, the accuracy of the surgical guides is extremely important, this subject being studied and debated intensely nowadays in the scientific literature. In the digital and analogous process of analysis, planning and production of the surgical guides errors can occur. Our review intend to synthesize the workflow steps and possible sources for inaccuracies.

\section{PRESENT OPTIONS FOR GUIDED SURGERY}

Guided implant surgery technique can be dynamic or static. The ability to adjust the implant planned position intraoperatively is an important advantage of the dynamic guided surgery (6). and 
use a computerized navigation system that assists real-time implant surgery. Although these methods are promising navigation systems, static guides are the most used method nowadays.

The statically guided surgical technique involves 3D data obtained from conical beam computer tomography (CBCT), surface optical scanning, computer aided design/computer assisted production (CAD/CAM), software for virtual implant planning and guide fabrication. The stabilization and support for fabricated surgical guide can be obtained on teeth, mucosa or bone, mini-implants, screws or pins (7). Once the guide is stabilized, the location of the drill holes and the drilling protocol is planned, which may include the use of the guide only for the pilot mill or for the partial or total use of the entire drill sequence. The implant insertion can be performed without a surgical guide or through the guide, in a fully guided approach.

\section{STEPS IN THE DIGITAL WORKFLOW}

The digital workflow can be divided into six steps, each one being able to produce deviations from the virtual plane - the correct execution of each stage needs to be carefully checked (8). It is worth noting that this process may also involve a combination of analogous and digital steps. In addition, there may be variations in the digital workflow in different softs of design.

\section{Evaluation of the patient}

During the patient's assessment, the complete aesthetic and functional analysis should include the following data: dental-periodontal status/evaluation of the existing dentures in the case of completely edentulous patients, initial radiographic evaluation that can be performed on two-dimensional X-rays for evaluation of the quantity and quality of the bone and the need for grafting, occlusal assessment essential for optimal functional integration of the future restoration. The amplitude of the mouth opening should be checked, as guided surgery requires additional access, especially in the posterior areas. The aesthetic evaluation is also made, the restauration design must provide the appropriate support for the lips and the optimum visibility white/pink, so that possible procedures for bone reduction or augmentation will be required.

\section{Data acquisition}

Evaluation using conventional 2D X-rays often provides misdiagnosis data for correct implant placement. Data collection includes Cone Beam CT (CBCT) recording and surface optical scanning. CBCT offers accurate linear measurements at low radiation dose compared to conventional radiation, allowing three-dimensional visualization of the alveolar ridge, identifying anatomical structures and pathologies. He is imported in the design soft for planning the intervention and it became mandatory even in seemingly simple cases (9).

A CBCT record can be obtained with or without an x-ray guide in dentate patients. For the edentate patient, the method of choice is the dual scan technique, but in the present techniques of direct mucosa scanning are also explored (10).

The limitations of CBCT in the case of total edentate are the contrast and distortion of the soft tissues. Distortion may be caused by patient's movement during recording or by artifacts (11) caused by high density materials such as composite fillings, metal restorations and implants. Distortion affects the quality of the image and therefore influences the accuracy of the guided surgery. Also, the use of different recording techniques with or without markers, can modify the radiological final result (12). The inaccuracy of the CBCT records in the soft tissue and teeth can be compensated by performing an optical surface scan, which records the surface of the teeth and the contour of the soft tissues. Any modification of the soft and hard tissues should be avoided after the scan, as the adaptation of the surgical guide will be compromised. Scanning can be created by direct or indirect methods. In the indirect method, the patient's dental print or cast model is scanned using a laboratory scanner. In the direct method, the intraoral scanner is used to record the area of interest of the patient's dental arch. Each arch should be scanned individually and then together in the occlusion. CBCT data is saved in Digital Imaging Communication in Medicine (DICOM) format and surface optical scanning is saved and transferred to Standard Tessellation Language file (STL). In addition, new CBCTs have the ability to overlap facial photos with CBCTs.

\section{Data manipulation}

The data imported into the planning software of digital implant are subject of the virtual segmentation and orientation of the DICOM file, the identification of the panoramic curve, spotting the pathway of the inferior alveolar nerve and the fusion of CBCT data with surface scanning.

One role of the segmentation of the raw data is differentiation and coloring of anatomical struc- 
tures and areas of interest. In addition, it reduces image distortion caused by reflection of metal surfaces and motion artifacts. The first stage of segmentation is to obtain the corresponding density threshold (gray value threshold) to clearly visualize the hard tissues (bone and teeth), manual configuration being the preferred method (13). Any portion of the scan (known as voxel) at a selected threshold, with the same or greater density, will be visible in the analyzed area. Threshold selection is subjective, and may be affected by bone irregularities. Gray thresholds can also influence 3D reconstruction and adaptation of surgical guides (14).

For the orientation and definition of the panoramic curve, the reconstructed volume rendered must be correctly oriented in the 3 planes, after which the panoramic arch must be defined.

The nerve path tracking tool of the design soft detect the lower alveolar canal by placing points along its path, which automatically are merged ensuring the visualization of the nerve path.

In the final stage, CBCT and surface data files are combined by selecting identical anatomical landmarks of tooth surfaces or radiological markers. A possible source of error may be the misalignment between the DICOM and STL datasets.

\section{Virtual planning of the implant insertion}

The design of the future prosthesis is based on a physical scanned model or a virtual modeling. The prosthesis wax up prefiguration (analogous or virtual) can be used to manufacture a provisional or definitive individual prosthesis. The prosthetic space must be judiciously evaluated during the projection of the restoration design.

In the virtual implant planning step, the implant type and size can be selected from the implant library provided by the software. The position of the implant and the insertion axis are adjusted according to the available bone supply. In the case of multiple implants, a parallelization tool can be used. Most systems offer the option to set a safety limit around and between implants, the system will warn the user if these limits are violated. At this stage, the possibility of a flapless approach or any need for bone augmentation is considered. To ensure a proper emergency profile and an access hole with optimal positioning during the planning, virtual attachments can be inserted at the implant level.

Once the virtual plan is completed, the user can design the surgical guide, including the type of support (tooth, tissue, bone or any combination). Surgical guide support is recommended to be done on a minimum of two teeth (15). The size of the sleeve (length and diameter) and the height (the distance between the sleeve and the implant platform) may vary between different systems, depending on the location of the implant and the planning requirements. In general, decreasing the height of the sleeve and using a shorter implant can reduce the angular deviation of the guided placement of the implant (15). In addition, this will allow the physician to perform the guiding operation in cases with limited mouth opening. The fully guided protocol will provide information on the optimal implantation and depth sequence at the level of an immediate prefabricated provisional prosthesis. The concept of "stackable guides" was introduced to reduce the surgical time and to improve the quality of the total provisional prosthesis in cases where the rehabilitation is performed exclusively by implant. A basic guide is arranged using the occlusal or anatomical landmarks and can serve as a bone reduction guide. The implant insertion guide is superimposed over the base guide to perform guided implant placement. Finally, the restoration of the arch morphology is done with a prefabricated provisional prosthesis with predefined holes.

After the planning phase is completed, the guides and designed prostheses are exported in an STL format for manufacturing. A detailed report is generated, which includes the drilling protocol corresponding to the implant type and the prosthetic components.

\section{Production of the guide and the prosthesis}

Surgical guides and prostheses can be manufactured using conventional methods or $\mathrm{CAD} / \mathrm{CAM}$ methods. The digital methods for the manufacture of the surgical guide, the models of the two arches and the prostheses can be additive (printing) or subtractive (milling). Printing involves the use of a 3D printer that cures photosensitive resin into the layers under the action of a light beam or laser, to generate the surgical guide and the stereolithographic models of the arches. After the surgical guide is printed, the metal sleeves specific to the implant system are incorporated. CAM milling systems offer many material options to produce temporary, final or abutment prostheses.

\section{Surgery}

Before the surgery, the correct installation and placement of the surgical guide must be checked. The surgical protocol is applied, which includes the size of the implant and the drilling sequence. Guide kits are specific to each implant system. 
The surgical guide can block sufficient irrigation of the prosthetic field and therefore more heat is released (16). However, the heat induced additionally is within acceptable limits of the temperature threshold. External irrigation is recommended to be done by pumping up and down, reducing the risk of overheating the bone during the guided drilling process (17).

\section{ACCURACY OF GUIDED IMPLANT SURGERY}

To evaluate the accuracy of implant surgery, preoperative and postoperative CBCT overlap or pre and post intervention models overlapping are used (18). Although guided surgery has become predictable, deviations will always exist between the virtual plane and the technical execution.

In 2018, the consensus publication of the International Team for Implantology (19) evaluated the accuracy of computer-assisted static implant surgery deviations of $1.2 \mathrm{~mm}$ at the mid-crest point, $1.4 \mathrm{~mm}$ at the apical point, $3.5^{\circ}$ angulation, $0.2 \mathrm{~mm}$ at the coronal depth and $0.5 \mathrm{~mm}$ were reported at the apical depth. Considering these data it is recommended that a safety margin of $2 \mathrm{~mm}$ is always respected.

\section{Type of surgical guide support}

Guided surgeries are more accurate in partially edentulous patients than in fully edentulous patients (19). The guides supported by the teeth are considered the most accurate, followed by the guides supported on the mucosa, those supported on the bone being considered the least accurate $(7,20)$. Factors that may influence the accuracy of mucosal supported guides are: bone density, mucosal thickness, local anesthesia (which causes tissue inflammation) and smoking (due to increased mucosal thickness) $(21,22,23)$. This is why mini-implants, fixing screws and stabilization pins are recommended to improve accuracy $(7,24)$ in three to four fixing points (19).

\section{Location of implant insertion}

The scientific literature reports about the accuracy of jaw-guided operations towards the maxilla or mandible remains controversial, with studies showing no difference $(7,25)$, others that show that the guided surgery in the mandible is more accurate $(24,26)$, while others indicate greater accuracy of guided surgery in the upper jaw $(4,27)$.

\section{Factors related to surgery}

The clinician's experience influence in the final result is controversial. Some studies conclusions
(28) show that the experience in conventional implant placement is an essential condition for the guided surgery good prognostic, but the results of the experienced surgeons were quantified in studies in the dynamically guided surgery (28), especially in terms of implant depth accuracy (29). Guided surgery has a positive correlation with reduced surgical complications (30).

The advantage of the flapless technique is the reduction of postoperative discomfort, surgical time, postoperative bleeding, and healing period (31) but sufficient keratinized tissue is needed providing low visibility (32). The flap technique improves intraoperative control but increases patient discomfort $(6,33)$. Pins fixing (25) and mucosal resilience (34) affects the stability and accuracy of mucosal support guides. Fully guided implant placement without flap removal with fixing screws is the most correct approach (24). The bone support guides require extensive flap reflection, which may interfere with the positioning of the guide; this may be an explanation for reduced accuracy (35).

Eccentric drilling through the sleeve has a substantial effect on accuracy, especially in the rear areas with limited access or limited mouth opening (36). Fully guided surgery is more accurate than a partially guided one $(7,24)$, but even if it has a higher accuracy and effectiveness, guided implant surgery is a technique that demands maximum exigency and it does not lack complications. Therefore, the idea that it requires less preparation is far from being true (37). However, the limited scientific evidence available suggests that guided placement of implants ensures a higher survival rate and overlying restorations, compared to conventional protocols with an equivalent clinical complication rate (38).

\section{CONCLUSION}

Guided surgery is a predictable technique if it is based on an accurate analysis and planning, the training of the surgeon and the fully guided implant placement without flap removal with fixing screws are the most important factors for a good result.

\section{Acknowledgement}

In this article, all the authors have equal contribution with the first author. 


\section{REFERENCES}

1. Jacobs R, Adriansens A, Naert I et al. Predictability of reformatted computed tomography for preoperative planning of endosseous implants. Dentomaxillofac Radiol, 1999; 28:37-41.

2. Tardieu PB, Vrielinck L, Escolano E et al. Computer-assisted implant placement: Scan template, simplant, surgiguide, and safe system. Int J Periodontics Restorative Dent, 2007; 27(2):141-149.

3. Kamposiora P, Papavasiliou G, Madianos P. Presentation of two cases of immediate restoration of implants in the esthetic region, using facilitate software and guides with stereolithographic model surgery prior to patient surgery. J Prosthodont, 2012; 21:130-137.

4. Amorfini L, Storelli S, Romeo E. Rehabilitation of a dentate mandible requiring a full arch rehabilitation. Immediate loading of a fixed complete denture on 8 implants placed with a bone-supported surgical computer-planned guide. A case report. J Oral Implantol Spec No. 2011; 37:106-113.

5. Rosenfeld AL, Mandelaris GA, Tardieu PB. Prosthetically directed implant placement using computer software to ensure precise placement and predictable prosthetic outcomes. Part 1: Diagnostics, imaging, and collaborative accountability. Int $\mathrm{J}$ Periodontics Restorative Dent, 2006; 26:215-221.

6. Jung RE, Schneider D, Ganeles J et al. Computer technology applications in surgical implant dentistry: A systematic review. Int J Oral Maxillofac Implants, 2009; 24:92-109.

7. Tahmaseb A, Wismeijer D, Coucke W et al. Computer technology applications in surgical implant dentistry: A systematic review. Int $J$ Oral Maxillofac Implants, 2014; 29:25-42.

8. Al Yafi F, Camenisch B, Al-Sabbagh M. Is Digital Guided Implant Surgery Accurate and Reliable? Dent Clin North Am, 2019; 63:381-397.

9. Omami G1, Al Yafi F. Should Cone Beam Computed Tomography Be Routinely Obtained in Implant Planning? Dent Clin North Am, 2019; 63:363-379.

10. Oh JH, An X, Jeong SM et al. Digital workflow for computer-guided implant surgery in edentulous patients: A case report. J Oral Maxillofac Surg, 2017; 75:2541-9.

11. Tadinada A, Jalali E, Jadhav A et al. Artifacts in cone beam computed tomography image volumes: An illustrative depiction. J Mass Dent Soc, 2015; 64:12-5.

12. Mohagheghi S, Ahmadian A, Yaghoobee S. Accuracy assessment of a marker-free method for registration of CT and stereo images applied in image-guided implantology: a phantom study. J Craniomaxillofac Surg 2014; 42:1977-84.

13. Flugge T, Derksen W, Te Poel J et al. Registration of cone beam computed tomography $\mathrm{V}$ data and intraoral surface scans $-\mathrm{a}$ prerequisite for guided implant surgery with CAD/CAM drilling guides. Clin Oral Implants Res, 2017; 28:1113-8.

14. Nackaerts O, Depypere M, Zhang G et al. Segmentation of trabecular jaw bone on cone beam CT datasets. Clin Implant Dent Relat Res. 2015; 17(6):1082-91.

15. Arisan V, Karabuda ZC, Ozdemir T. Accuracy of two stereolithographic guide systems for computer-aided implant placement: A computed tomography-based clinical comparative study. J Periodontol. 2010; 81:43-51.

16. El Kholy K, Janner SFM, Schimmel M et al. The influence of guided sleeve height, drilling distance, and drilling key length on the accuracy of static computer assisted implant surgery. Clin Implant Dent Relat Res. 2019; 21:101-7.

17. Markovic A, Lazic Z, Misic T et al. Effect of surgical drill guide and irrigans temperature on thermal bone changes during drilling implant sites - thermographic analysis on bovine ribs. Vojnosanit Pregl. 2016; 73(8):744-50.

18. Jeong SM, Yoo JH, Fang $Y$ et al. The effect of guided flapless implant procedure on heat generation from implant drilling. J Craniomaxillofac Surg. 2014; 42:725-9.

19. Komiyama A, Pettersson A, Hultin M et al. Virtually planned and template-guided implant surgery: An experimental model matching approach. Clin Oral Implants Res. 2011; 22:308-13.
20. Tahmaseb A, Wu V, Wismeijer D et al. The accuracy of static computer-aided implant surgery: A systematic review and metaanalysis. Clin Oral Implants Res. 2018; 29 (Suppl 16):416-35.

21. Raico Gallardo YN, da Silva-Olivio IRT, Mukai E et al. Accuracy comparison of guided surgery for dental implants according to the tissue of support: A systematic review and meta-analysis. Clin Oral Implants Res. 2017; 28:602-12.

22. Seo C, Juodzbalys G. Accuracy of guided surgery via stereolithographic mucosa-supported surgical guide in implant surgery for edentulous patient: a systematic review. J Oral Maxillofac Res. 2018; 9(1): e1.

23. Cassetta M, Pompa G, Di Carlo S et al. The influence of smoking and surgical echnique on the accuracy of mucosa-supported stereolithographic surgical guide in complete edentulous upper jaws. Eur Rev Med Pharmacol Sci, 2012; 16:1546-53.

24. Sun Y, Luebbers HT, Agbaje JO et al. Accuracy of dental implant placement using CBCT-derived mucosa-supported stereolithographic template. Clin Implant Dent Relat Res. 2015; 17:862-70.

25. Zhou W, Liu Z, Song L et al. Clinical factors affecting the accuracy of guided implant surgery - a systematic review and meta-analysis. J Evid Based Dent Prac. 2018; 18:28-40.

26. Ozan O, Orhan K, Turkyilmaz I. Correlation between bone density and angular deviation of implants placed using CT-generated surgical guides. J Craniofac Surg. 2011; 22:1755-61.

27. Cassetta M, Giansanti M, Di Mambro A et al. Accuracy of positioning of implants inserted using a mucosa-supported stereolithographic surgical guide in the edentulous maxilla and mandible. Int $J$ Oral Maxillofac Implants. 2014; 29:1071-8.

28. Cassetta M, Bellardini M. How much does experience in guided implant surgery play a role in accuracy? A randomized controlled pilot study. Int J Oral Maxillofac Surg. 2017; 46:922-30.

29. Rungcharassaeng K, Caruso JM, Kan JY et al. Accuracy of computerguided surgery: A comparison of operator experience. J Prosthet Dent. 2015; 114:407-13.

30. Cassetta M, Stefanelli LV, Giansanti M et al. Depth deviation and occurrence of early surgical complications or unexpected events using a single stereolithographic surgi-guide. Int J Oral Maxillofac Surg, 2011; 40:1377-87.

31. Jung RE, Schneider D, Ganeles J et al. Computer technology applications in surgical implant dentistry: A systematic review. Int $\mathrm{J}$ Oral Maxillofac Implants, 2009; 24(Suppl):92-109.

32. Abboud M, Wahl G, Calvo-Guirado JL et al. Application and success of two stereolithographic surgical guide systems for implant placement with immediate loading. Int J Oral Maxillofac Implants, 2012; 27:634-43.

33. Rosenfeld AL, Mandelaris GA, Tardieu PB. Prosthetically directed implant placement using computer software to ensure precise placement and predictable prosthetic outcomes. Part 2: rapid-prototype medical modeling and stereolithographic drilling guides requiring bone exposure. Int J Periodontics Restorative Dent. 2006; 26:347-53.

34. Vasak C, Watzak G, Gahleitner A et al. Computed tomography-based evaluation of template (NobelGuide)-guided implant positions: A prospective radiological study. Clin Oral Implants Res. 2011; 22:1157-63.

35. Lal K, White GS, Morea DN et al. Use of stereolithographic templates for surgical and prosthodontic implant planning and placement. Part I. The concept. J Prosthodont. 2006; 15:51-8.

36. Laederach V, Mukaddam K, Payer M et al. Deviations of different systems for guided implant surgery. Clin Oral Implants Res. 2017; 28:1147-51.

37. Sicilia A, Botticelli D, Working G. Computer-guided implant therapy and soft- and hard-tissue aspects. The third EAO consensus conference. Clin Oral Implants Res. 2012; 23(Suppl 6):157-61.

38. Joda T, Derksen W, Wittneben JG et al. Static computer-aided implant surgery (s-CAIS) analysing patient-reported outcome measures (PROMs), economics and surgical complications: A systematic review. Clin Oral Implants Res. 2018; 29(Suppl 16):359-73. 